\title{
Singular Spectrum Analysis (SSA) Based Tremor Supression In Micro-Surgery
}

\author{
Ibrahim Nasir Said", Abubakar Aliyu Badawi**, Farouk Nasir Said ${ }^{* * *}$ \\ ${ }^{*}$ Newcastle University, UK \\ ** Bayero University, Kano
}

DOI: 10.29322/IJSRP.12.01.2022.p12120

http://dx.doi.org/10.29322/IJSRP.12.01.2022.p12120

\begin{abstract}
Tremor as a result of unstable nature of human organ is one of the main problems in micro surgery due to its effects in generating undesirable surgical outcomes. The unwanted movement impedes microsurgery and lead to poor performance. Various techniques were proposed to improve the effect of tremor to achieve high level of precision. In this work, SSA algorithm is developed to suppress the physiological tremor present in the hands of surgeon. The technique decomposes the time domain signal into different singular spectrum (interpretable components) domain via the singular value decomposition (SVD), in which primary signal such as trends and oscillatory components can be recognized, isolated from the tremor and regrouped in a linear fashion, and finally time domain signals reconstructed. The developed algorithm promises potential for tremor suppression in realtime. By choosing a group of specific decomposed signals based on their eigenvalues and spectral range, both the primary and tremor motions can be reconstructed accurately. The algorithm shows the tremor signal can be estimated from the total motion with an accuracy greater than $89 \%$.
\end{abstract}

Index Terms- Micro-surgery, Singular Spectrum Analysis (SSA), Tremor, Eigen values and Eigen vectors

\section{INTRODUCTION}

$\mathrm{R}$ hythmic and involuntary movement of body part is described as physiological tremor. Tremor is a random event and generates a rough periodic sinusoidal component that occurred at a single dominant frequency. The intensity of the tremors is relatively small in magnitude and present in all humans [2]. Micromanipulation task which require precise accuracy and manual positioning is affected by tremor. Abnormal muscle activation pattern is the ultimate reason for tremor in humans originates from the central nervous system [1]. While sleeping and anesthesia, tremor disappears and increases with stress and fatigue [5].

Precision of typical micro-meters is of great importance because, high accuracy is needed in microsurgery. Hand-held surgical instruments are highly and usually affected by erroneous involuntary movement. So, the imprecision as a result of surgeon's hand caused by wrist, fingers and pronation-supination which may lead to bad performance of surgeon has to be overcome [1]. The amplitude of physiological tremor is about 50 $\mu m$ and lies in a frequency band of $8-12 \mathrm{~Hz}$ represented approximately by a sinusoidal movement [1][3]. This tremor leads to complicating many delicate surgical procedures which eventually result in some interference to be practically impossible. It is obvious that, the factor limiting the number of professional microsurgeons is the manual accuracy. Hand stability degrades with increasing age and hence, further escalates the situation. Fatigue, alcohol and caffeine consumption and many more factors escalates manual stability of microsurgeons even in the youthful age of the surgeons [4].

A well-recognized technique for noise suppression and cancellation called singular spectrum analysis (SSA) is used to overcome the limitations of other algorithms. SSA is widely used in biomedical signal processing with many aims and objectives. Among the objectives include removing noise elements, extracting the voluntary signal, forecasting, change point detection or eliminating high-amplitude artifacts. The principle of SSA algorithm is based on singular value decomposition (SVD) of a covariance matrix generated from the time series.

The elimination of constituent in a signal and their grouping is based on identifying various trend on the eigenvalue spectra criterion or based on specific statistics of the extracted component. The removal of a frequency component is associated to the component related with its corresponding eigenvalues. For instance, removing noise interference in a signal is usually addressed by eliminating components related to small eigenvalues [7].

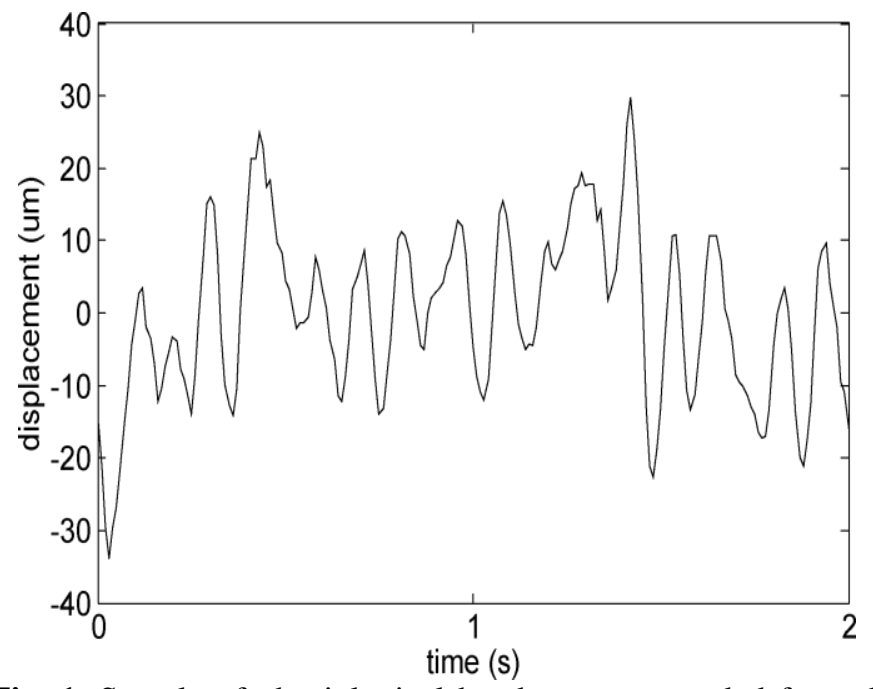

Fig. 1. Sample of physiological hand tremor recorded from the tip of a hand-held microsurgical instrument measured in $\mu$ [3]

It is important to note that the model allow to decompose a time series into different number of components which implied 
different frequency bands of the original signal. Comparison of these methods and performance analysis will be evaluated from the results obtained to ascertain the suitability of each algorithm. The performance of SSA is evaluated with a very high accuracy.

\section{SINGULAR SPECTRUM ANALYSIS (SSA)}

The first literature of SSA can be traced in a publication by Broomhead and King in 1986. Since then, SSA has received a significant attention by researchers in literature [9].

Singular spectrum is a term derived from the word "spectral" meaning eigenvalue. It involves the decomposition of a given matrix into a sub-set of spectrum (eigenvalues). If the determinant of the eigenmatrix denoted by $(A-\lambda \mathrm{I})$ is zero, then the eigenvalues represented by the symbol $\lambda$ are numbers that make the eigenmatrix singular [6], given that the symbol $\mathrm{I}$ is the identity matrix. SSA is a well-established and a general algorithm used for time series analysis and forecasting [10][12]. The technique is suitable and employed in removal of noise component present in a noisy signal or to learn the spectral profile of the signal [7].

SSA aims at decomposing a time series into a sum of various interpretable components. These interpretable components can be classified as: slowly varying trend, oscillatory components and a 'structureless' noise interferences [7]. Due to the non-parametric nature of the algorithm, it has time series applications ranging from social sciences, mathematics economics, oceanography and biomedical signal processing due to their non-parametric model nature [7][12].

Basic SSA is an algorithm that basically comprises of two complementary stages, i.e. decomposition and reconstruction. Each of the complementary stages, there are two sub-stages, which are discussed below.

\section{A. Decomposition}

The aim of this stage is to embed a real valued time series $x(t)=$ $x_{1}, x_{2}, \ldots, x_{N}$ of length $N$ into multi-dimensional Euclidean space. The multi-dimensional vector space then finds a subspace corresponding to the required component. The two main stages of decomposition are namely; Embedding followed by singular value decomposition (SVD). The stages of decomposition are explained below:

\section{i. Embedding}

Embedding is the process of mapping that transforms the nonzero one-dimensional time series $x=x_{1}, x_{2}, \ldots, x_{N}$ of length $N$ into multi-dimensional sequence of lagged vectors, $\boldsymbol{X}=$ $\left[X_{1}, X_{2}, \ldots, X_{K}\right]$. With the size of the lagged vector $L$, it leads to forming $\mathrm{K}$ lagged vectors and is given by: $K=N-L+1$. The value of the window length $\mathrm{L}$ is within $2 \leq L \leq N-1$. The outcome of the decomposition is given by $\boldsymbol{X}$ called trajectory matrix with a dimension of $L \times K$ matrix. The elements in ascending diagonals of the trajectory matrix $X$ are identical and sometimes known as Henkel matrix $\boldsymbol{X}$. In time series analysis, embedding is a standard procedure [14].

$$
\boldsymbol{X}=\left[X_{1}: X_{2}: \ldots X_{K}\right]
$$

The $L \times K$ trajectory matrix is given by:

$$
\boldsymbol{X}=\left[\begin{array}{cccc}
x_{1} & x_{2} & \cdots & x_{K} \\
x_{2} & x_{3} & \cdots & x_{K+1} \\
\vdots & \vdots & \ddots & \vdots \\
x_{L} & x_{L+1} & \cdots & x_{N}
\end{array}\right]
$$

ii. Singular Value Decompostion (SVD)

The second stage of a basic SSA is singular value decomposition (SVD). It refers to the transformation of the sample covariance matrix $\boldsymbol{S}=\boldsymbol{X} \boldsymbol{X}^{\boldsymbol{T}}$, so that $\boldsymbol{S}$ can be expressed as $\boldsymbol{S}=\boldsymbol{U} \boldsymbol{\Lambda} \boldsymbol{V}^{\boldsymbol{T}}$. It provides the related subspace modeled via the eigenvalues matrix $\Lambda=\left[\lambda_{1}, \lambda_{2}, \ldots, \lambda_{L}\right]$ to be sorted in decreasing order of magnitude and their respective eigenvectors $U=\left[U_{1}, U_{2}, \ldots, U_{L}\right]$ are also sorted similarly [11][9]. $\boldsymbol{V}$ is referred as the factor vector and can be calculated using the relation below:

$$
V_{i}=\frac{X^{T} U_{i}}{\sqrt{\lambda_{i}}} \quad \text { where }(i=1,2, \ldots \ldots, L)
$$

Therefore, the trajectory matrix can be expressed as a sum of rank-one $\boldsymbol{L}$ matrices after performing the SVD as:

$$
\boldsymbol{X}=\left[X_{1}+X_{2}+\cdots+X_{L}\right]
$$

Where $X_{i}=\sqrt{\lambda_{i}} U_{i} V_{i}$ and it is called the $i_{t h}$ eigentriple group which forms an elementary matrix $X_{i}$.

\section{B. Reconstruction}

Reconstruction is the second stage of SSA and sometimes called estimation stage. The time series is reconstructed from the selected elementary matrices. It is achieved in two main substages namely:

\section{i. $\quad$ Eigentriple Grouping}

The eigentriple grouping basically identify the suitable window length of the signal. This step corresponds to splitting the elementary matrix into several groups and adding the matrices within each group [11]. In other words, the elementary matrices are respectively grouped together depending on the whether the decomposed signal is oscillatory signal of varying period, slow varying trend or structure-less noise interferences. The primary or dominant signals are represented by the first groups of the eigentriples and are those with high eigenvalues. In distinction, the noise signal is represented by the last few eigentriples with low eigenvalues. Therefore, to extract the desired signal, the first set of the elementary matrices with the higher eigentriple can be grouped together [9][11]. Mathematically,

$$
X_{I}=X_{I_{1}}+X_{I_{2}}+X_{I_{3}}+\ldots X_{I_{m}}
$$

$X_{I}$ is the trajectory matrix of the desired signal, whereas the matrices to the right-hand side of the equation are its constituents elementary matrices. 


$$
\boldsymbol{X}=\left[\begin{array}{cccc}
\hat{x}_{11} & \hat{x}_{12} & \ldots & x_{1 K} \\
\hat{x}_{21} & \hat{x}_{22} & \cdots & \hat{x}_{2 K} \\
\vdots & \vdots & \ddots & \vdots \\
\hat{x}_{L 1} & \hat{x}_{L 2} & \cdots & \hat{x}_{L K}
\end{array}\right]
$$

The above process of grouping sets of eigentriples $I_{1}, I_{2}, \ldots, I_{m}$ is called eigentriple grouping

\section{ii. Diagonal Averaging}

After the trajectory matrix is computed as shown in equation 5 and 6, each matrix of the component would be transformed into a new time series from the subgroups of length $N$. The transformation of the elementary matrix $X_{I}$ by diagonal averaging yields the desired sub-series [9]. Diagonal averaging is applied to generate a new series such that the initial series is decomposed into sum of the reconstructed series. Hence, an accurate estimate of the sub-series of interest $\hat{X}_{N}$ is obtained by averaging it cross diagonally [9][11] . The sub-series of interest can be depicted by:

Where

$$
\hat{X}_{N}=\hat{x}_{1}, \hat{x}_{2}, \hat{x}_{3}, \ldots, \hat{x}_{N}
$$

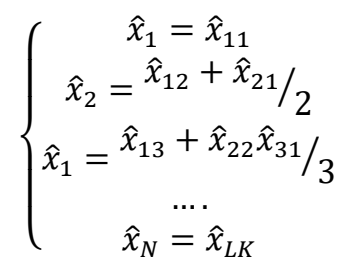

\section{RESULT}

The simulation of SSA algorithm was performed in MATLAB to become familiar with the algorithm parameters and its operation. Similarly, the voluntary (primary) signal was a sinusoidal wave of frequency $0.5 \mathrm{~Hz}$ and amplitude of 2 . The length of the generated time series was chosen to be the length of the generated time series i.e. $\mathrm{N}=1751$. For the signal time analysis, in order to separate the trends or oscillation components a value of window length $\mathrm{L}$ was taken as approximately N/4=500 as recommended value and should be large enough but not greater than $N / 2$. If $L$ is too large, there will be larger oscillations to be resolved and too few observations from which to estimate the covariance matrix of the $\mathrm{L}$ variables. Noise signal of amplitude 0.05 was randomly generated as the first component of the tremor to the clean sine signal. The second tremor component was generated with different frequencies at different point in the time series. The generated distorted signal i.e. noise (random and multiple frequency generated) and the voluntary signal was the input to the filter. Four SSA stages were applied to the signal to separate the main components (desired) signal from the noise (tremor). The project uses 4 modes to analyze and separate the components of the signal. The result of the four stages is given below:

\section{Embedding}

Mapping of the one-directional time series to multi-directional sequence of lagged vector is the first stage. The trajectory matrix known as Hankel matrix is a matrix with $L \times K$ dimension from the original time series. The time series of the signal is shown in fig. 2 below:
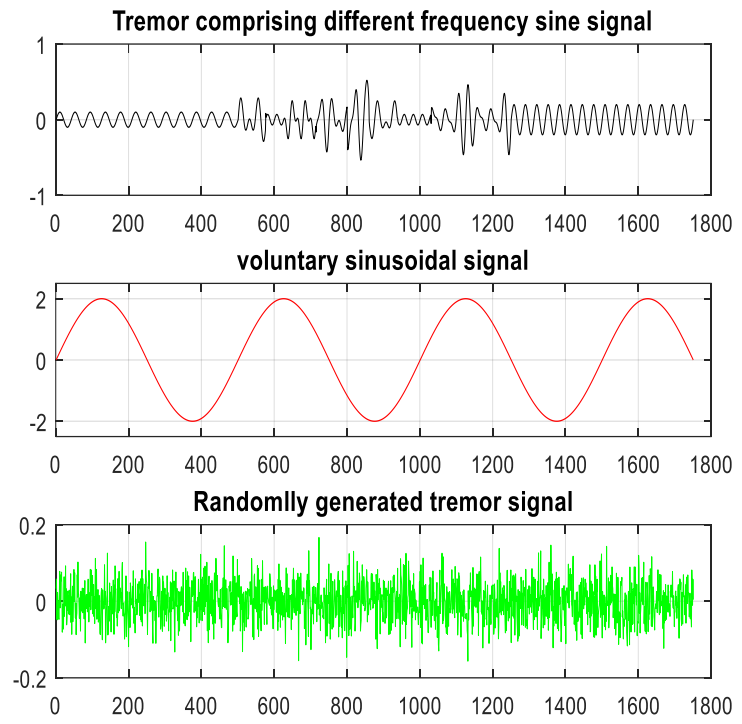

Fig. 2. Tremor and voluntary signals

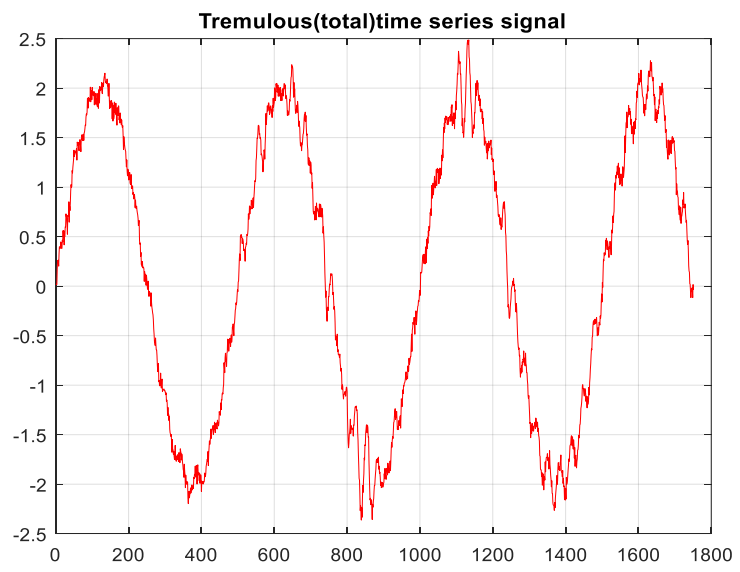

Fig. 3. Tremulous (whole) time series signal

\section{$S V D$}

The noisy signal was decomposed into components, as shown in fig. 5 below. A covariance matrix was constructed by performing matrix operations and which is required for the computations of eigenvalues and eigenvectors. The eigenvalues were presented along the main diagonal of a square matrix and the method is called singular value decomposition (SVD). Their corresponding eigenvectors are required to compute the principal components were also presented as they represent the axes of projection in a square matrix. 

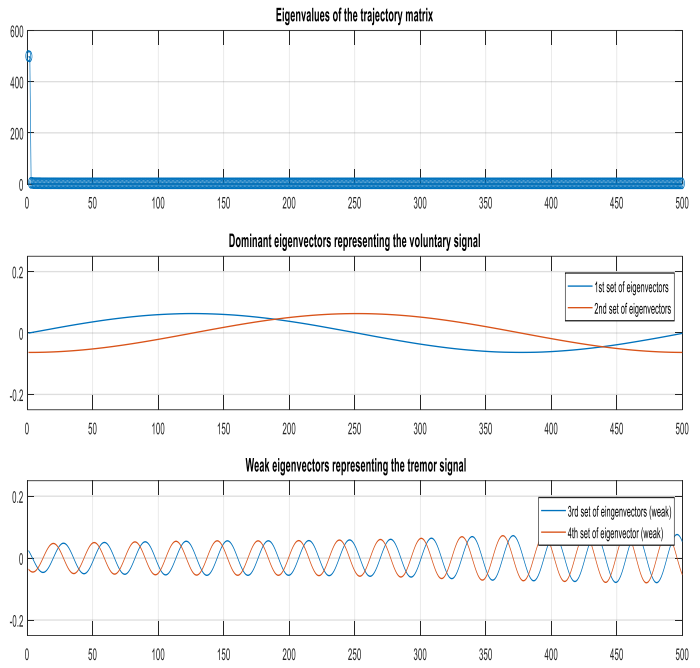

Fig. 4. Eigenvalues and Eigenvectors

The result of the modes according to singular values were in decreasing order. So, fig. 5 shows decomposition result of the first 4 modes. Fig. 4 shows the eiganvalues from a decomposed signal corrupted with tremor arranged in decreasing order of magnitude.

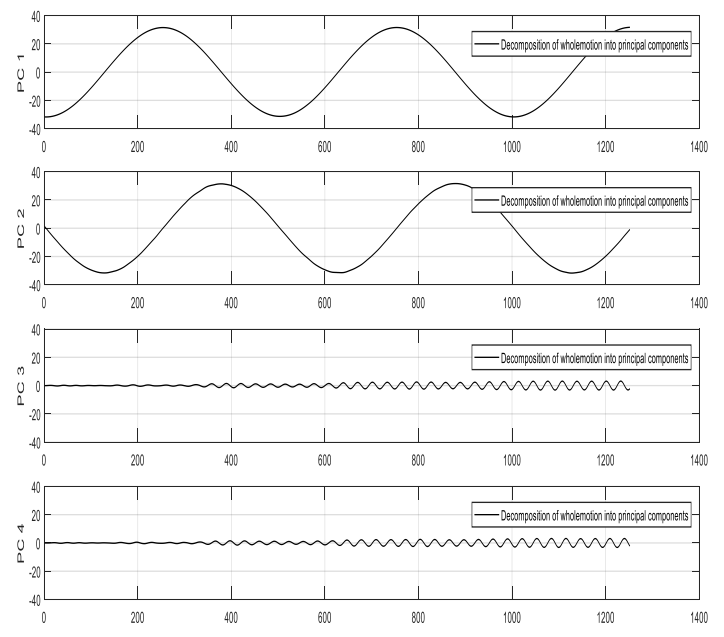

Fig. 5. Principal components after performing the SVD

The figure above shows how a basic SSA successfully decomposes the whole motion into slow varying voluntary motion, oscillating tremor motion with different frequencies and random noise. The high frequency oscillation was represented by the first eigenvectors considering the successive elements of the eigenvectors over time. The lower frequency oscillation components represent the tremor (noise) of the time series.

\section{Grouping}

Based on the selection on the most dominant pairs of the eigenvalues, the grouping was performed.

Principal components were computed by projecting the trajectory matrix onto the eigenvectors. The principal components corresponds to the most dominated eigenvectors comprises of the clean signal, while the tremor signal corresponds to the component with lower amplitudes.

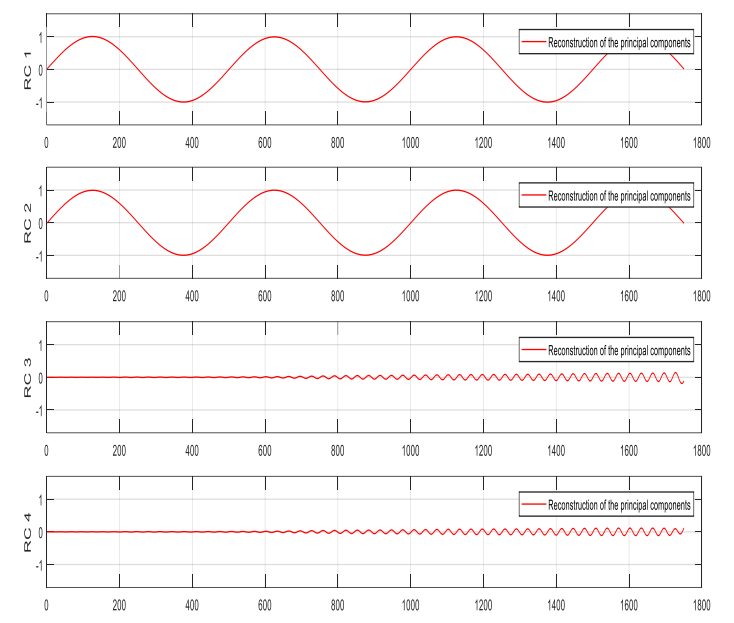

Fig. 6. Reconstructed components using diagonal averaging

The algorithm up till this point, the signal was represented in eigen domain, Therefore, principal components are incomparable to the original time series.

The components for reconstruction of the time series was properly chosen based on the singular spectrum appearance. The reconstructed component was selected as the first two oscillatory component corresponds to the important oscillations. In disparity, the eigenvalues associated with the last two components are mostly located as the tremor signal of the singular spectra.

The output is the singular spectrum of the original time series which is a column vector that represents the eigenvalues.

Lastly, figure below depicts a comparison of the tremulous (noisy) signal with the reconstructed signal (filtered). It can be concluded from the comparison of the signals that, a considerable tremor signal was filtered from the noisy signal by choosing the leading 2 eigentriples with window length $\mathrm{L}=500$. Moreover, the findings discovered that the de-noised signal is similar the clean (original) signal. However, it can be concluded that SSA readily remove tremor and reconstruct periodic components from tremulous time series. 

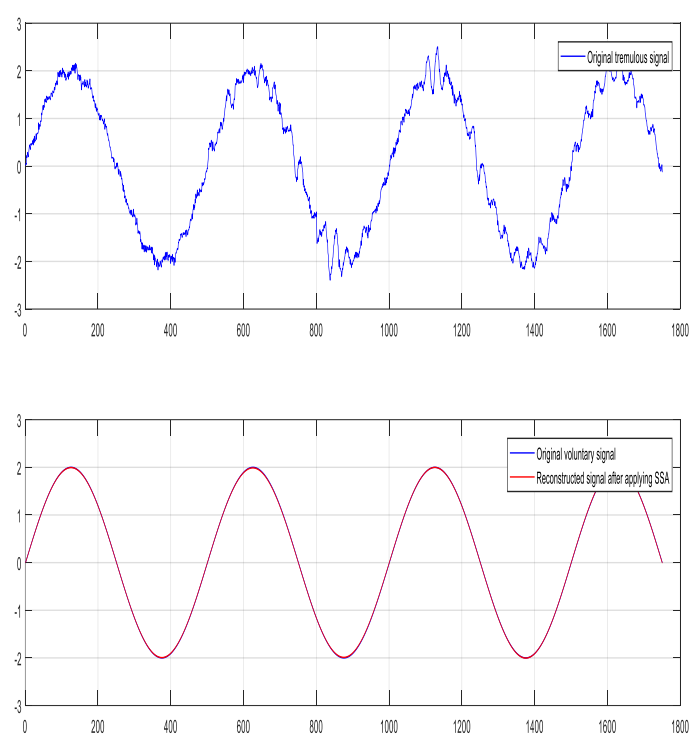

Fig. 7. Tremulous and comparison between the original voluntary signal with the reconstructed signal using SSA

\section{DISCUSSION}

It can be concluded that even without knowing the prior information about the generated data, the first two modes reconstructions corresponding to the first two singular values represent the main (voluntary) components of the time series. The accuracy and performance of the decomposition of the signal is determined by the value of the window length $\mathrm{L}$ only of the SSA algorithm.

The algorithm generates a trajectory matrix from the original time series by sliding a window of length $\mathrm{L}$. It is always important using the auxiliary information about the original time series for the choice of the values of windows length and eigenvalues/singular values. In SSA algorithm, the window length $\mathrm{L}$ is the only parameter in the decomposition stage. The windows length is the only parameter of the algorithm which determines the accuracy of the decomposition of a signal. If the original time series has a specific component with integer period, then the value of $\mathrm{L}$ should be proportional to the period. It was suggested that the time series of length $\mathrm{N}$, the choice of the window length $\mathrm{L}$ is $\mathrm{N} / 4$ is an appropriate and common in practice [9][13]. Nina recommends that the windows length $\mathrm{L}$ should be large enough but should not be larger than half of the series length. To attain a minimum error based on optimal selection of $\mathrm{L}$ is problem dependent i.e. depends on the type of noise. Therefore, Nina suggests that the window length L should be half the value of the time series in most cases [9]. Despite different attempts that have been employed to obtain result with minimum error, there is no universal rule for obtaining optimal selection of the window length and eigen values. Furthermore, Golyandina and Zhigljavsky point out that that for signal extraction of voluntary motion from noise, the significance in selection of the value of $\mathrm{L}$ is diminished [9]. In conclusion, the windows length of SSA should be sufficiently large. In particular, the value of $\mathrm{L}$ has to be large enough so that each $\mathrm{L}$ lagged vector incorporates an essential part of the of the initial time series. But, if $L$ is too large, this will leave too few observations from which to estimate the covariance matrix of the $L$ variables. Generally, too large values of $L$ induce longer period oscillations to be resolved.

Using the recommendations set above, the value of window length was chosen to be 500. Based on the windows length selected, the trajectory matrix is approximated using SVD. By performing matrix operations, the covariance matrix is constructed which is required for computing the eigenvalues and eigenvectors. We have 500 eigentriples ordered by their contribution in the decomposition. The refined series is obtained by identifying and grouping of the eigentriples of the SVD of the trajectory matrix of the original time series that corresponds to the voluntary signal. Furthermore, an appropriate choice of $L$ and $r$ (eigenvalues) leads to a perfect analysis and separability between time series components. Choosing a very small value of L could lead to a mix-up between components which otherwise would be interpretable and therefore weak separability.

Another important aspect of consideration is the grouping technique used in this project. The grouping technique is simplistic approach based on the eigen-triple for separating the decomposed components after grouping similar components together.

Despite all the advantages of the SSA, one of the drawbacks of this algorithm is that, the method is mostly developed for realvalued signals. The result indicates the potential of the SSA as a valid algorithm for tremor suppression.

\section{PERFORMANCE ANALYSIS}

We calculate the tremor estimation percentage accuracy (\%) to measure and ascertain the performance of SSA. For validation, the voluntary motion from the recorded total signal (whole motion) was pre-filtered using 5th-order zero-lag Butterworth filter with a $5 \mathrm{~Hz}$ cut off frequency. This filtering technique known as forward-backward obtains the voluntary (primary) motion without any phase delay. Thus, it serves as an ideal fact for performance validation. Forward- backward filtering technique is only possible offline and hence cannot be implemented in real- time applications [5][8]. The actual tremor signal is then obtained by deducting this pre-filtered voluntary motion from the total motion. Therefore, the percentage accuracy of the SSA can be calculated as:

$$
\operatorname{Accuracy}(\%)=\frac{\operatorname{RMS}\left(x_{k}\right)-\operatorname{RMS}\left(e_{k}\right)}{R M S\left(x_{k}\right)} \times 100 \%
$$

Where:

And

$$
\operatorname{RMS}\left(x_{k}\right)=\sqrt{\frac{1}{N} \sum_{K=1}^{N} x_{k}^{2}}
$$

$$
R M S\left(e_{k}\right)=\sqrt{\frac{1}{N} \sum_{K=1}^{N}\left(x_{k}-\widehat{x_{k}}\right)^{2}}
$$


The variables $x_{k}, \widehat{x_{k}}$ and $e_{k}$ represents the actual pre-filtered tremor signal, estimated tremor signal and the error between the actual and estimated tremor signal respectively. The average tremor estimation accuracy is $\mathbf{8 9 . 7 6 \%}$, which is required for the tremor compensation as shown in fig. 8 below:

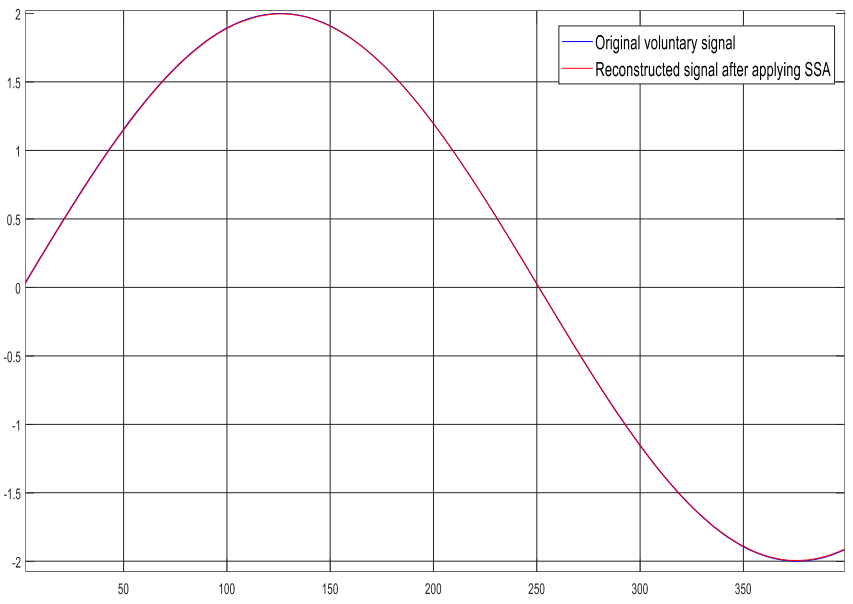

Fig.8. Accuracy of SSA algorithm i.e. comparison between original voluntary signal and reconstructed signal by SSA algorithm

Table I: SSA Characteristics of SSA in relation to tremor suppression

\begin{tabular}{|l|l|}
\hline Characteristics & SSA tremor detection \\
\hline Able to detect sinusoidal tremor & Yes \\
\hline Able to detect sinusoidal tremor & Yes \\
\hline $\begin{array}{l}\text { Able to detect multiple dominant } \\
\text { frequencies }\end{array}$ & Yes \\
\hline Presence of delay & No \\
\hline Presence of high adaptation time & No \\
\hline Accuracy & High \\
\hline
\end{tabular}

\section{CONSLUSION}

The project presented an investigation of the SSA algorithm which shows it can be a promising method for physiological tremor estimation in real-time, improving upon previous adaptive filter-based. The algorithm yields accurate and robust tremor amplitude and frequency estimation. It is important to select a suitable tremor filtering algorithm which suppress its effects, yet maintains the structure of the signal. SSA is a powerful model free SVD based method and effective for tremor reduction.

\section{References}

[1] Kumar, A., Kumar, S., Kaushik, A., Kumar, A. and Saini, J.S., 2020. Real time estimation and suppression of hand tremor for surgical robotic applications. Microsystem Technologies, pp.1-7.

[2] Veluvolu, K.C. and Ang, W.T., 2011. Estimation of physiological tremor from accelerometers for real-time applications. Sensors, 11(3), pp.30203036.

[3] Riviere, C. N., Gangloff, J., \& De Mathelin, M. (2006). Robotic compensation of biological motion to enhance surgical accuracy. Proceedings of the IEEE, 94(9), 1705-1716.
[4] Adhikari, K., Tatinati, S., Veluvolu, K. C., \& Nazarpour, K. (2015). Improvement in modelling of physiological tremor by inclusion of grip force in quaternion weighted fourier linear combiner.

[5] Adhikari, K., Tatinati, S., Ang, W.T., Veluvolu, K.C. and Nazarpour, K., 2016. A quaternion weighted Fourier linear combiner for modeling physiological tremor. IEEE Transactions on Biomedical Engineering, 63(11), pp.2336-2346.

[6] Eldwaik, O. and Li, F.F., 2017. Microphone wind noise reduction using singular spectrum analysis techniques. Proceedings of the Institute of Acoustics, Vol. 39 Pt. 1.

[7] Tomé, A.M., Malafaia, D., Teixeira, A.R. and Lang, E.W., 2018. On the use of Singular Spectrum Analysis. arXiv preprint arXiv:1807.10679.

[8] Georgescu, V. and Delureanu, S.M., 2015, August. Fuzzy-valued and complex-valued time series analysis using multivariate and complex extensions to singular spectrum analysis. In 2015 IEEE International Conference on Fuzzy Systems (FUZZ-IEEE) (pp. 1-8). IEEE.

[9] Golyandina, N. and Zhigljavsky, A., 2013. Singular Spectrum Analysis for time series. Springer Science \& Business Media.

[10] Wang, Y., Tatinati, S., Adhikari, K., Huang, L., Nazarpour, K., Ang, W.T. and Veluvolu, K.C., 2018. Multi-step prediction of physiological tremor with random quaternion neurons for surgical robotics applications. IEEE Access, 6, pp.42216-42226.

[11] Hassani, H., 2007. Singular spectrum analysis: methodology and comparison.

[12] Adhikari, K., Tatinati, S., Veluvolu, K.C., Chambers, J.A. and Nazarpour, K., 2017. Real-time Physiological Hand Tremor Estimation using Recursive Singular Spectrum Analysis. In 39th Annual International Conference on Engineering in Medicine and Biology Society (EMBC). Newcastle University.

[13] Bó, A.P.L., 2010. Compensation active de tremblements pathologiques des membres supérieurs via la stimulation électrique fonctionnelle (Doctoral dissertation, Montpellier 2).

[14] Gonzalez, J.G., Heredia, E.A., Rahman, T., Barner, K.E. and Arce, G.R., 2000. Optimal digital filtering for tremor suppression. IEEE Transactions on Biomedical Engineering, 47(5), pp.664-673. 